\title{
Fault detection for Modular Multilevel Converters Based on Sliding Mode Observer
}

\author{
Shuai Shao, Patrick W. Wheeler, Member, IEEE, Jon C. Clare, Senior Member, IEEE, and \\ Alan J. Watson, Member, IEEE
}

\begin{abstract}
This letter presents a fault detection method for modular multilevel converters (MMC) which is capable of locating a faulty semiconductor switching device in the circuit. The proposed fault detection method is based on a sliding mode observer (SMO) and a switching model of a half-bridge, the approach taken is to conjecture the location of fault, modify the SMO accordingly and then compare the observed and measured states to verify, or otherwise, the assumption. This technique requires no additional measurement elements and can easily be implemented in a DSP or micro-controller. The operation and robustness of the fault detection technique are confirmed by simulation results for the fault condition of a semiconductor switching device appearing as an open-circuit.
\end{abstract}

Index Terms-Fault detection, modular multilevel converter, sliding mode observer, switching model.

\section{INTRODUCTION}

$\mathbf{T}$ HE modular multilevel converter (MMC) has drawn considerable interest, as it offers very attractive features [1]-[4].

- Modular construction with scalable, manufacturable, standardised cells (half-bridge).

- Submodules are fed by floating DC capacitors, no multipulse transformer is required.

- High power and high voltage capability, extendable by adding additional cells.

- Flexible control of the voltage level and simple realization of redundancy if required.

Fault detection is an important issue for a MMC. When an open-circuit fault occurs, the output voltage and current are distorted, moreover, the voltages of the DC floating capacitors will keep increasing, leading to further, vast destruction. Therefore, it is vital to locate the fault after its occurrence and take measures such as bypassing the faulty cell to reconfigure the MMC.

Given the large numbers of identical cells (half-bridge) and the symmetrical structure of the converter, the process of fault location in a MMC is challenging if significant extra cost is to be avoided. An effective but inefficient way to detect faults is to add additional sensors to each semiconductor switching device [5], to each cell [6], or to use a gate drive module capable of detecting faults and providing feedback [7]. These

Manuscript received June 26, 2012; revised November 6, 2012.

The authors are with the Power Electronics, Machines and Control Group, School of Electrical and Electronic Engineering, University of Nottingham, Nottingham, NG7 2RD, U.K. (email: eexss21@nottingham.ac.uk; Pat.Wheeler@nottingham.ac.uk; Jon.Clare@nottingham.ac.uk; Alan.watson@nottingham.ac.uk). additional sensors and signals increase not only the cost but also the implementation complexity. Some conventional fault detection methods for voltage source converters (VSCs), such as the calculation of the output current trajectory using Park's Vector [8] [9] or comparison of the actual AC voltage and the reference quantity [10] are however not suitable for a MMC, as there is not enough information present to locate the fault.

Fault diagnosis methods for a cascaded H-bridge (CHB) are applicable to a MMC because of the similar structure. Reference [11] presented a detection approach for a CHB multilevel converter, which analyses magnitude of the switching frequency component $\left(v_{s}\right)$ of the output phase voltage: $v_{s}$ becomes significantly larger after the occurrence of a fault due to the imbalanced cancellation of the switching frequency harmonics. The faulty cell can be located according to the angle of $v_{s}$ [11]. However, the faulty switching device cannot be located, and it is complex to implement and easy to get the wrong diagnosis in transient operation. In [12] the authors proposed an artificial intelligence (AI) based algorithm to detect the fault of a $\mathrm{CHB}$, the major drawbacks are the accuracy (only $76 \%$ in some cases) and the long training time required for the circuit and all the fault scenarios.

This paper proposes a sliding mode observer (SMO) based fault detection method for a MMC. The method uses the converter arm currents and the cell capacitor voltages as the inputs, which are already available as measurement inputs to the control system, no additional sensors are required. Using this method not only the faulty cell, but also the faulty switching device can be located. Moreover, inherited from the easy implementation and robustness of the SMO [13][15], this method has good immunity to conditions such as transient operation, degradation of capacitance over time and measurement inaccuracies.

\section{SWITCHING MODEL OF A HALF-BRIDGE}

A half-bridge (Fig. 1) is the basic cell of a MMC. In order to diagnose an open-circuit fault, it is essential to identify the characteristics of a half-bridge as observed from its DC-side and AC-side both in normal and fault conditions.

$g_{1}$ and $g_{2}$ in Fig. 1 are the gate signals for the switches, and are complementary. When the gate signal is 1 , the corresponding switch turns on; when it is 0 , the corresponding switch turns off.

The analysis assumes ideal devices and instantaneous commutation. The fault detection method is however robust against non-ideal device characteristics. This is verified in the all of 


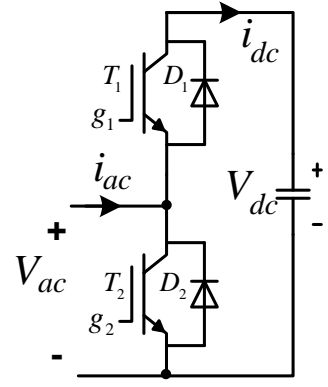

(a)

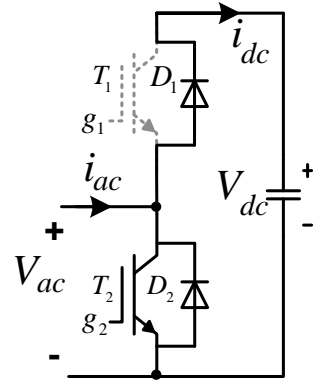

(b)
Fig. 1: Switching model of half-bridge. (a)Normal condition. (b)Fault condition (an open-circuit fault at $T_{1}$ ).

the simulation results where generous values of $5 \mathrm{~V}$ and $1 \mu \mathrm{s}$ are included for the device voltage drop and dead-time delay respectively.

1) Normal (fault-free) condition: As shown in Fig. 1(a), when $g_{1}=1, g_{2}=0, T_{1}$ is on and $T_{2}$ is off, thus $V_{a c}=$ $V_{d c}, i_{d c}=i_{a c}$; alternatively, when $g_{1}=0, g_{2}=1, V_{a c}=$ $0, i_{d c}=0$. Therefore, the relationship between the AC-side and DC-side voltages and currents can be calculated as

$$
\left\{\begin{array}{l}
V_{a c}=S \cdot V_{d c} \\
i_{d c}=S \cdot i_{a c}
\end{array}\right.
$$

where $S$ is the switching state given by Table I.

TABLE I: Switching state $S$ in normal condition

\begin{tabular}{|c|c|}
\hline $\mathrm{S}$ & Driving signals \\
\hline 1 & $g_{1}=1, g_{2}=0$ \\
\hline 0 & $g_{1}=0, g_{2}=1$ \\
\hline
\end{tabular}

2) Fault condition: In the fault condition (one open-circuit fault of the switch), the switching models can still be described as (1), but the switching states $S$ have to be modified.

Consider the half-bridge with an open circuit fault at $T_{1}$, as shown in Fig. 1(b). When $g_{1}=1, i_{a c}<0, i_{a c}$ is forced to go through $D_{2}$ instead of $T_{1}$ as the result of the open-circuit fault. Thus, the switching state $S$ should be changed from 1 to 0 . For all other conditions, the half-bridge operates just as normal.

When the open-circuit fault occurs on $T_{2}$, the switching state can be modified in a similar way. Table II demonstrates the modifications of switching states of a faulty half-bridge.

TABLE II: Switching state $S$ in fault condition

\begin{tabular}{|c|c|c|c|}
\hline \multirow{2}{*}{$\begin{array}{c}\text { Location of } \\
\text { the fault }\end{array}$} & Condition & \multicolumn{2}{|c|}{ Switching State } \\
\cline { 3 - 4 } & & Normal & Fault \\
\hline \multirow{2}{*}{$T_{1}$} & $g_{1}=1, i_{a c}<0$ & 1 & $S_{F}=0$ \\
\cline { 2 - 4 } & Other conditions & $\mathrm{S}$ & $S_{F}=S$ \\
\hline \multirow{2}{*}{$T_{2}$} & $g_{2}=1, i_{a c}>0$ & 0 & $S_{F}=1$ \\
\cline { 2 - 4 } & Other conditions & $\mathrm{S}$ & $S_{F}=S$ \\
\hline
\end{tabular}

\section{SLIDING MOde ObSERVER}

An observer is a contrivance designed from a real system, generally in the same mathematical form as the original system, so as to estimate its internal state [13] [16]. The SMO uses high-gain feedback in the observer vector (normally in the form of a high frequency switching function, for example the saturation function of an observed-measured error, as (3) and (4) present) to force the observed output to converge to the actual output [13] [15]. The SMO offers desirable features such as robustness to parameter uncertainty and insensitivity to measurement noise [13]-[15], [17]. With simple realization, the SMO can be implemented in the FPGA [16] [17].

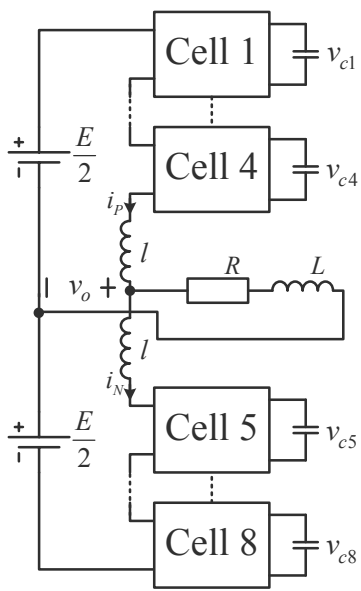

(a)

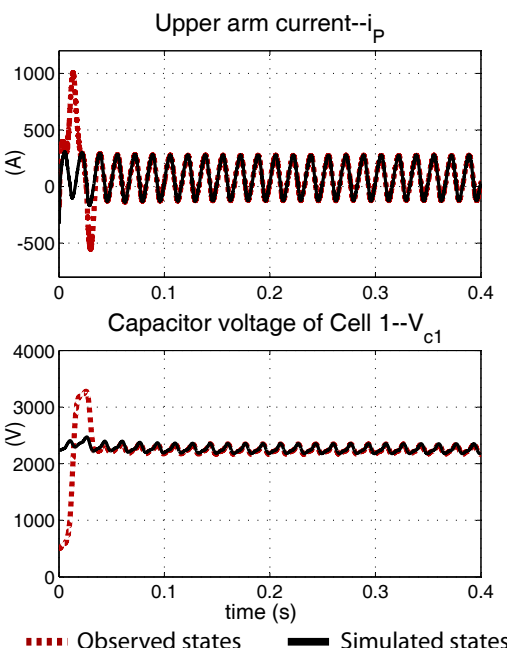

(b)
Fig. 2: (a) An eight cell MMC circuit (each cell represents a half-bridge). (b) SMO simulated results in normal conditions.

Based on [16] [18], the SMO can be developed for a single phase eight cell MMC as shown in Fig. 2(a). The equations which characterize the upper arm can be expressed as

$$
\begin{aligned}
& \frac{d i_{P}}{d t}=-\frac{1}{l}\left(S_{1} v_{c 1}+S_{2} v_{c 2}+S_{3} v_{c 3}+S_{4} v_{c 4}+v_{o}-\frac{E}{2}\right) \\
& \frac{d v_{c i}}{d t}=\frac{1}{C} S_{i} i_{P}(i=1,2,3,4),
\end{aligned}
$$

where $l$ is the arm inductance, as shown in Fig. 2(a), $C$ is the DC-capacitance, $v_{c 1}, \cdots, v_{c 4}$ are the capacitor voltages of cell 1 to cell 4 respectively, $S_{1}, \cdots, S_{4}$ are the corresponding switching states of the half-bridges given by Table I.

If $i_{P}$ and one of the capacitor voltages $\left(v_{c 1}, \cdots, v_{c 4}\right.$, we consider $v_{c 1}$ in this case) are selected to be observed, then the SMO equation is

$$
\begin{aligned}
\frac{d \hat{i}_{P}}{d t}= & -\frac{1}{l}\left(S_{1} \hat{v}_{c 1}+S_{2} v_{c 2}+S_{3} v_{c 3}+S_{4} v_{c 4}+\right. \\
& \left.v_{o}-\frac{E}{2}\right)-L_{1} \operatorname{sat}\left(\hat{i}_{P}-i_{P}\right) \\
\frac{d \hat{v}_{c 1}}{d t}= & \frac{1}{C} S_{1} \hat{i}_{P}-L_{2} \operatorname{sat}\left(-l S_{1} L_{1} \operatorname{sat}\left(\hat{i}_{P}-i_{P}\right)\right),
\end{aligned}
$$

where $\hat{i}_{P}$ and $\hat{v}_{c 1}$ denote the observed states associated with the actual states $i_{P}$ and $v_{c 1} ; L_{1} \operatorname{sat}\left(\hat{i}_{P}-i_{P}\right)$ and 
$L_{2} \operatorname{sat}\left(-l S_{1} L_{1} \operatorname{sat}\left(\hat{i}_{P}-i_{P}\right)\right)$ comprise the observer vector, whose derivations are detailed in [16] [18]; $L_{1}$ and $L_{2}$ are the observer gains (large constant, for example 20000) to guarantee the sliding mode; sat $(x)$ is the saturation function, which is defined as

$$
\operatorname{sat}(x)=\left\{\begin{array}{lr}
1 & x \geq 1 \\
x & -1<x<1 \\
-1 & x \leq-1
\end{array}\right.
$$

According to the analysis of [16], the system described in (3) is fully observable.

The MMC circuit is simulated using SIMULINK/PLECS. The observed and actual simulated states are shown in Fig. 2(b), from which one can see that $\hat{i}_{P}$ and $\hat{v}_{c 1}$ accurately match $i_{P}$ and $v_{c 1}$ respectively. The lower arm current and capacitor voltage can be observed in the same way.

\section{FAULT DETECTION ON A MMC USING SLIDING MODE OBSERVER}

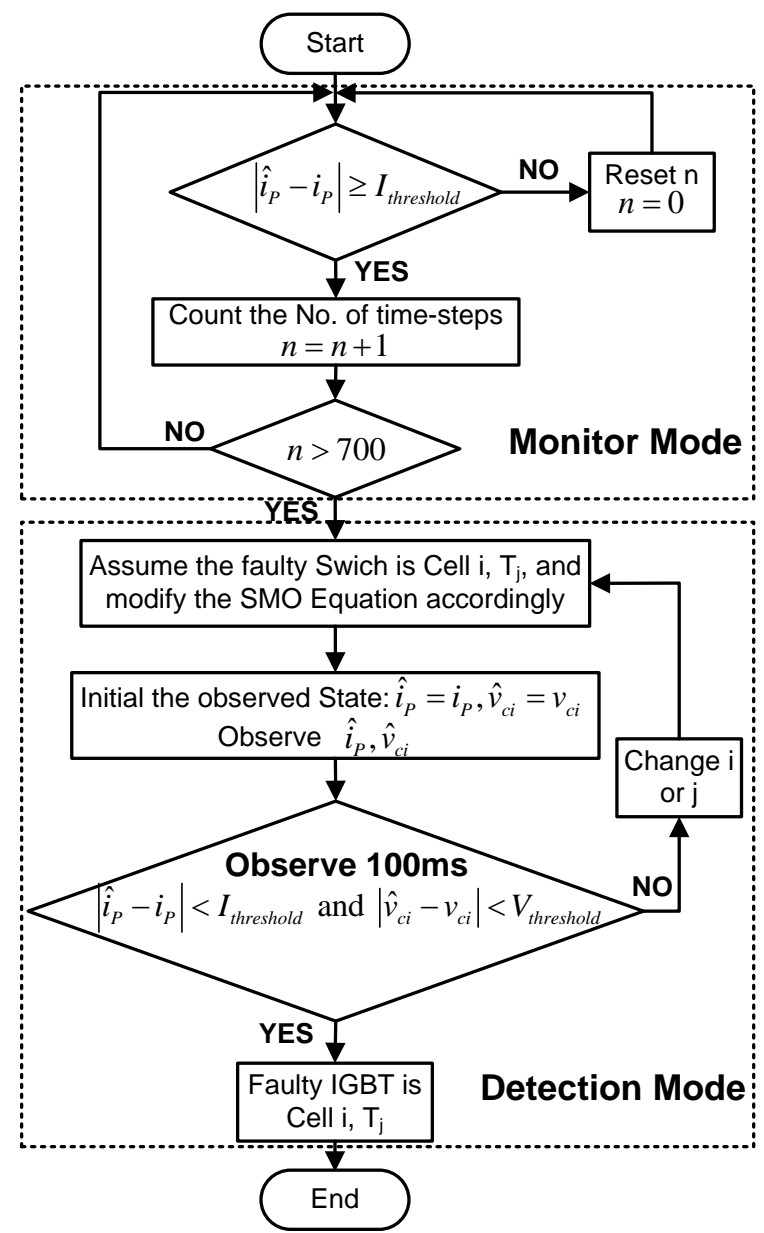

Fig. 3: Flow chart of the fault detection.

The basic idea of this detection method is to compare the observed state and the actual simulated state of the MMC, and if they are different for a predefined period of time, then a fault has occurred, and a procedure including assumption, modification and judgement to locate the open-circuit fault begins.
The fault detection systems consists of two modes, as specified in Fig. 3. The process of each mode is detailed as follows. Consider the upper arm of the MMC circuit in Fig. 2(a), and suppose that it initially operates in normal, steady conditions.

Monitor mode: The aim of this mode is to determine whether the MMC is operating normally. $i_{P}$ and one of the any capacitor voltages (for example $v_{c 1}$ ) are observed.

If $\left|\hat{i}_{P}-i_{P}\right| \geq I_{\text {Threshold }}$, where $I_{\text {threshold }}$ is the threshold value of current error, and it lasts for 700 time steps (2us per step), then an open-circuit fault has occurred. We denote this moment as $t_{0}$ and go to the Detection mode; else, repeat the Monitor mode. Note that the decision of fault occurrence is made only if the observed-simulated error $\left(\hat{i}_{P}-i_{P} \mid\right)$ lasts for a period of time (700 time-steps in this letter, empirical value), this is useful to prevent "false positives", as the measurement noise may also lead to $\left|\hat{i}_{P}-i_{P}\right| \geq I_{\text {Threshold }}$.

Detection mode: The aim of this mode is to locate the opencircuit after its occurrence.

D1. [Assumption and Modification] Set the assumed faulty switch as Cell $i(i=1,2,3,4), T_{j}(j=1,2)$, modify $S_{i}$ (replace $S$ with $S_{F}$ ) of SMO in (3) based on the Table II, set $\hat{i}_{P}\left(t_{0}\right)=i_{P}\left(t_{0}\right), \hat{v}_{c i}\left(t_{0}\right)=v_{c i}\left(t_{0}\right)$, and observe $i_{P}$ and $v_{c i}$. For example, if the assumed faulty switch is Cell $4, T_{1}$, then $i_{P}$ and $v_{c 4}$ are observed, and the observer equations are

$$
\begin{aligned}
\frac{d \hat{i}_{P}}{d t}= & -\frac{1}{l}\left(S_{4(F)} \hat{v}_{c 4}+S_{1} v_{c 1}+S_{2} v_{c 2}+S_{3} v_{c 3}+\right. \\
& \left.v_{o}-\frac{E}{2}\right)-L_{1} \operatorname{sat}\left(\hat{i}_{P}-i_{P}\right) \\
\frac{d \hat{v}_{c 4}}{d t}= & \frac{1}{C} S_{4(F)} \hat{i}_{P}-L_{2} \operatorname{sat}\left(-l S_{4(F)} L_{1} \operatorname{sat}\left(\hat{i}_{P}-i_{P}\right)\right) .
\end{aligned}
$$

D2. [Judgement] If $\left|\hat{i}_{P}-i_{P}\right|<I_{\text {Threshold }}$ and $\left|\hat{v}_{c i}-v_{c i}\right|$ $<V_{\text {Threshold, }}$, where $V_{\text {threshold }}$ is the threshold value of voltage error, and it lasts for $100 \mathrm{~ms}$ (empirical value), then the assumption is correct-the faulty switch is Cell $i(i=$ $1,2,3,4), T_{j}(j=1,2)$; else alter $i$ or $j$, go to $D l$ to try another switch.

Note that the threshold value ( $I_{\text {Threshold }}$ and $V_{\text {Threshold }}$ ) are chosen in such a way that the observed-simulated error $\left(\left|\hat{i}_{P}-i_{P}\right|\right.$ and $\left.\left|\hat{v}_{c i}-v_{c i}\right|\right)$ are significant enough to allow a decision to be made with low risk of "false positive". In this letter, $I_{\text {Threshold }}$ is the peak value of the arm current, and $V_{\text {Threshold }}$ is $1 / 10$ of the DC capacitor voltage.

The Detection mode can be executed in parallel for each cell, such that the open-circuit fault can be located within a very short time.

\section{Simulated Results}

Consider an open-circuit fault occurring at Cell $4, T_{1}$ at $t=0.1 s$, as illustrated in Fig. 4 (a).

Firstly, Fig. 4 (b) shows that if no fault detection algorithm is applied, the observed states cannot follow the actual simulated states after the occurrence of the open-circuit fault. One can see that $\hat{i}_{P}$ and $\hat{v}_{c 1}$ cannot track $i_{P}$ and $v_{c 1}$ after $t=0.1 s$. 


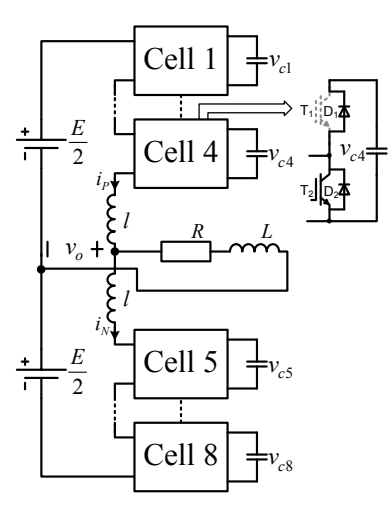

(a)

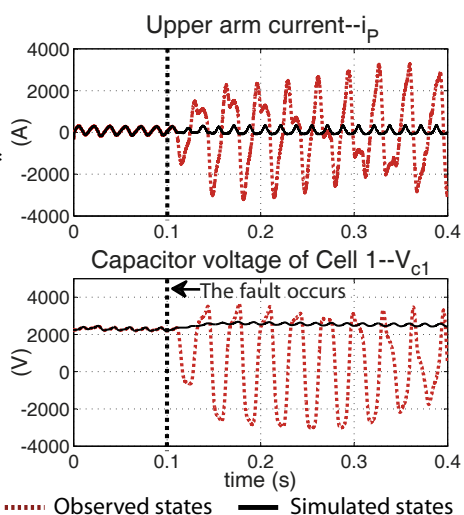

(b)
Fig. 4: (a)An open-circuit fault occurs at Cell 4,T $T_{1}$. (b) Observed and actual simulated states without using the fault detection algorithm.
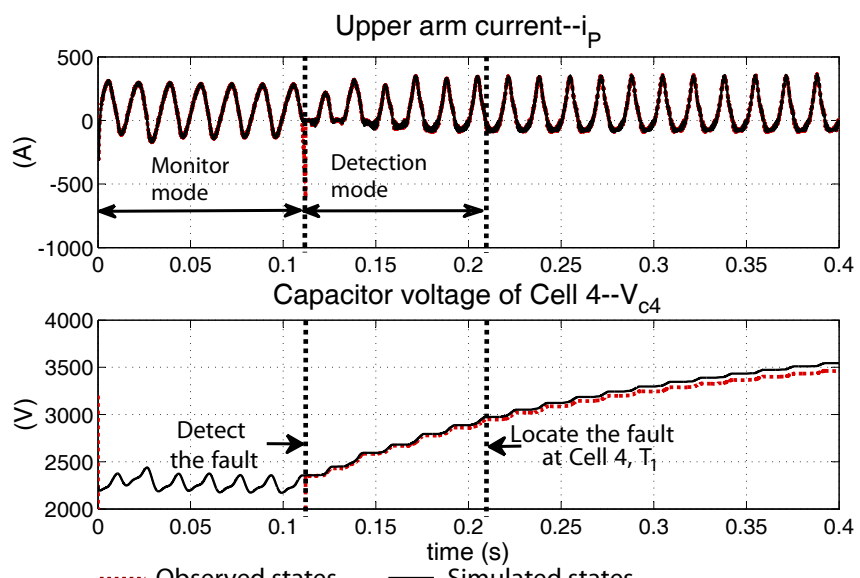

Fig. 5: Fault detection process: assume the faulty switch is Cell $4, T_{1}$.
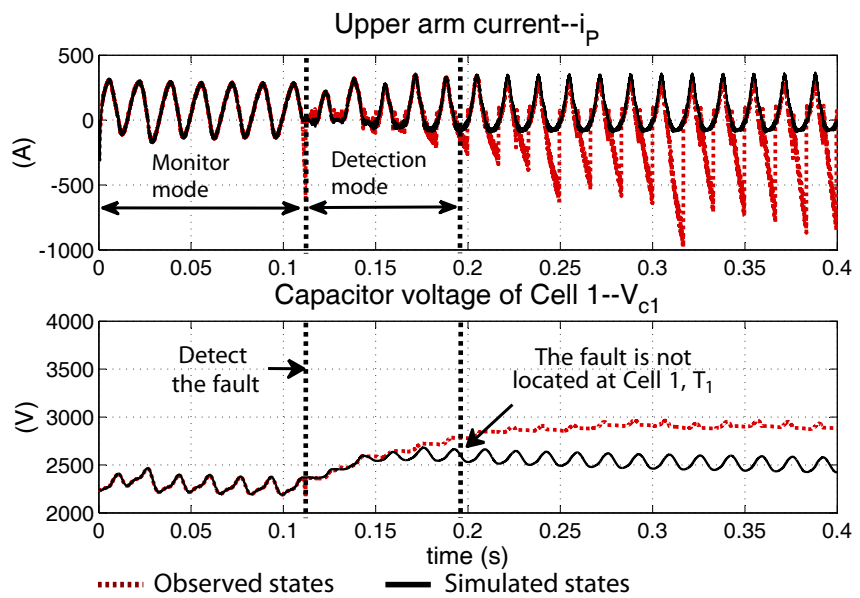

Fig. 6: Fault detection process: assume the faulty switch is Cell 1, $T_{1}$.

Secondly, Fig. 5 and Fig. 6 illustrate the process of the fault detection. In the monitor mode (0 to $0.1 s)$, the observed current $\hat{i}_{P}$ can follow the simulated current $i_{P}$, as shown in both

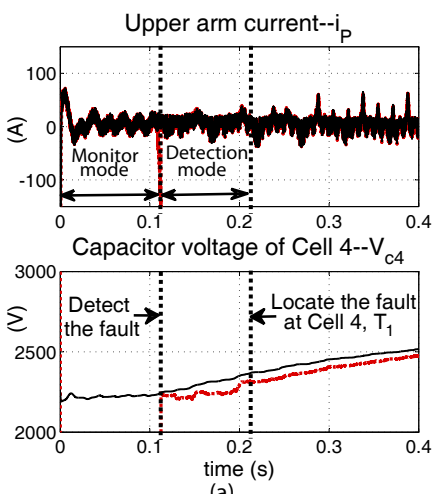

(a)

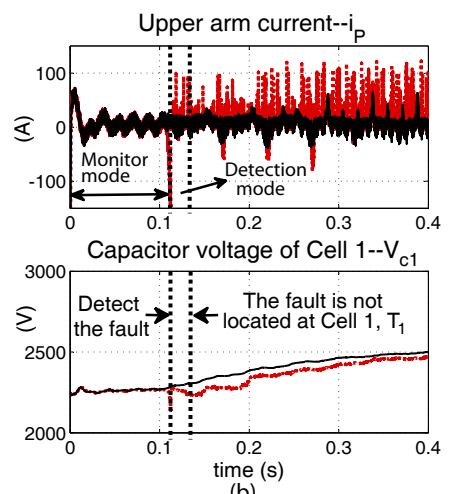

(b)
Fig. 7: Fault detection at the 5\% load condition.

Fig. 5 and Fig. 6. After $0.1 s$, the occurrence of the open-circuit fault is detected with the condition $\left|\hat{i}_{P}-i_{P}\right| \geq I_{\text {Threshold }}$, and the algorithm enters into the detection mode. In Fig. 5, the assumed the faulty switch is the actual one-Cell $4, T_{1}$. It can be seen that the observed states $\hat{i}_{P}$ and $\hat{v}_{c 4}$ can track $i_{P}$ and $v_{c 4}$ again. In Fig. 6, the assumed faulty switch is Cell $1, T_{1}$. The observed states $\hat{i}_{P}$ and $\hat{v}_{c 1}$ cannot track the simulated states $i_{P}$ and $v_{c 1}$ after the modification, and it can be judged that the faulty switch is not Cell $1, T_{1}$ as $\left|\hat{v}_{c 1}-v_{c 1}\right|>V_{\text {Threshold }}$ within $100 \mathrm{~ms}$.

Finally, the light load condition is considered. At the 5\% load, when the assumed faulty switch is the actual oneCell 4, $T_{1}$, the observed states can follow the actual states, as shown in Fig. 7 (a); when the assumed faulty switch is not the actual one, a significant difference between observed and simulated current $\left(\left|\hat{i}_{P}-i_{P}\right|>I_{\text {Threshold }}\right)$ is appeared soon after the algorithm enters into the Monitor Mode, as shown in Fig. 7 (b). The open-circuit fault can still be located at the light load condition.

\section{Robustness}

The proposed SMO-based fault detection method has immunity to the parameter uncertainty, measurement inaccuracy and imbalanced voltage. A simulation has been undertaken to demonstrate these properties, and part of the simulation parameters have been amended as follows to imitate the parameters and measurement errors and the imbalanced capacitor voltage. Other conditions are the same as the ones in Section V.

- Parameter uncertainty. Let the arm inductance used in the calculation be $110 \%$ of the actual value, and the DCcapacitance $120 \%$ of the actual value, namely, $l_{\text {cal }}=$ $1.1 l, C_{\text {cal }}=1.2 C$.

- Measurement inaccuracy. Add 2\% of the systematic error $\left(\Delta_{S}\right)$ and $10 \%$ of the random error $\left(\Delta_{R}\right)$ to the measured capacitor voltage, namely, $v_{c i \_c a l}=\left(1+\Delta_{S}+\Delta_{R}\right) \cdot v_{c i}$.

- Imbalanced capacitor voltage. Let $V_{c 1}=1.25 V_{c 4}$ before the occurrence of the open-circuit fault.

The results are illustrated in Fig. 8 and Fig. 9. In the monitor mode (0 to $0.1 s$ ), the observed current can still track the simulated current. One can also notice that the capacitor 

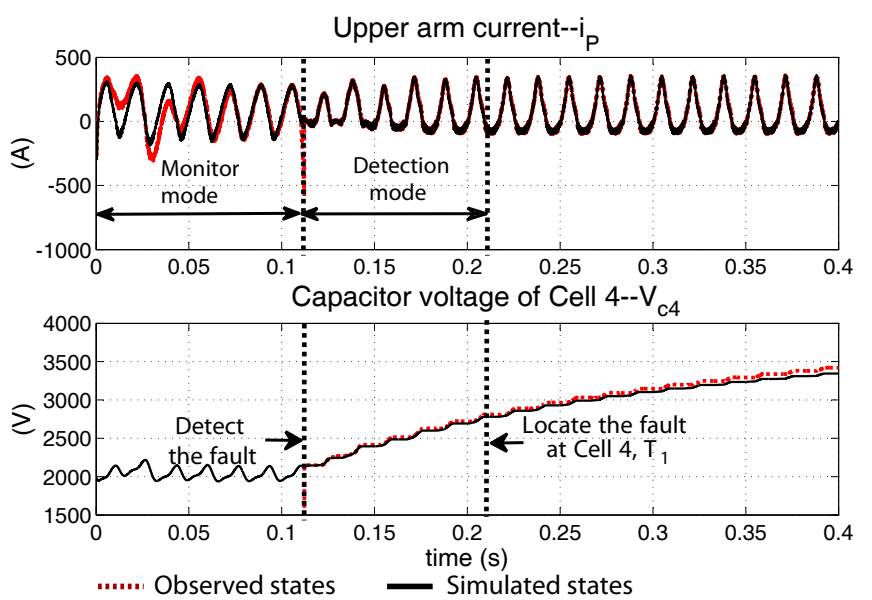

Fig. 8: Robustness test: assume the faulty switch is $C e l l 4, T_{1}$.
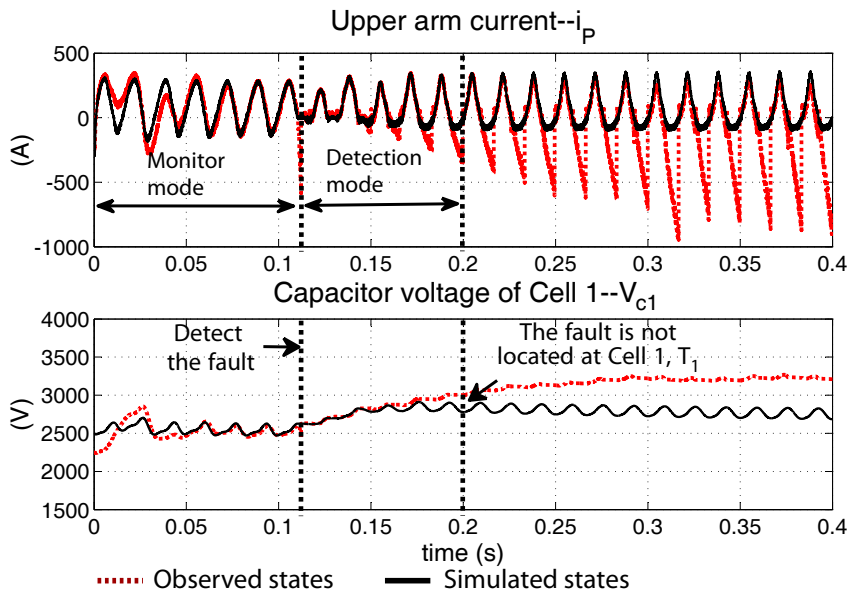

Fig. 9: Robustness test: assume the faulty switch is Cell $1, T_{1}$.

voltage is imbalanced during this period: $v_{c 1}$ in Fig. 9 is 1.25 times the value of $v_{c 4}$ in in Fig. 8. In regard to locating the fault (after $0.1 s$ ), when the assumed switch is the actual faulty one, the observed states $\hat{i}_{P}$ and $\hat{v}_{c 4}$ can track $i_{P}$ and $v_{c 4}$, as Fig. 8 presents; whereas the assumed switch is not the actual one, the observed $\hat{i}_{P}$ and $\hat{v}_{c 1}$ cannot match $i_{P}$ and $v_{c 1}$, as Fig. 9 illustrates. In the presence of parameter uncertainty and measurement noise and imbalanced capacitor voltage, the faulty switch can still be located.

\section{CONCLUSION}

This paper proposes a SMO-based fault detection method for a MMC. Simulations, which include device voltage drops and commutation delays, have shown that it is effective in locating the faulty switch and very robust against both parameter uncertainty and measurement error. This method is also independent of operation frequency of a MMC. With parallel execution, the open-circuit fault can be located within a very short time. The accuracy of the measurement may limit the application of this method-it is not able to locate the fault when the systematic measurement error is larger than $7 \%$.

Construction of experiments is currently underway, the SMO is going to be implemented in the ACTEL A3P400
FPGA, while the control system and the fault detection algorithm will be carried out in the TMS320C6713 DSP, this is similar to to the approaches taken in [17] and [18].

This method can also be used on any other multilevel half or full bridge converters.

\section{REFERENCES}

[1] A. Lesnicar and R. Marquardt, "An innovative modular multilevel converter topology suitable for a wide power range," in Power Tech Conference Proceedings, 2003 IEEE Bologna, vol. 3, june 2003, p. 6 pp. Vol.3.

[2] M. Hagiwara and H. Akagi, "Control and experiment of pulsewidthmodulated modular multilevel converters," Power Electronics, IEEE Transactions on, vol. 24, no. 7, pp. 1737 -1746, july 2009.

[3] G. Adam, O. Anaya-Lara, G. Burt, D. Telford, B. Williams, and J. McDonald, "Modular multilevel inverter: Pulse width modulation and capacitor balancing technique," Power Electronics, IET, vol. 3, no. 5, pp. $702-715$, september 2010.

[4] G. T. Son, H.-J. Lee, T. S. Nam, Y.-H. Chung, U.-H. Lee, S.-T. Baek, K. Hur, and J.-W. Park, "Design and control of a modular multilevel hvdc converter with redundant power modules for noninterruptible energy transfer," Power Delivery, IEEE Transactions on, vol. PP, no. 99, p. 1, 2012.

[5] F. Huang and F. Flett, "Igbt fault protection based on di/dt feedback control," in Power Electronics Specialists Conference, 2007. PESC 2007. IEEE, june 2007, pp. $1478-1484$.

[6] O.-S. Yu, N.-J. Park, and D.-S. Hyun, "A novel fault detection scheme for voltage fed pwm inverter," in IEEE Industrial Electronics, IECON 2006 - 32nd Annual Conference on, nov. 2006, pp. 2654 -2659.

[7] W. Song and A. Huang, "Fault-tolerant design and control strategy for cascaded h-bridge multilevel converter-based statcom," Industrial Electronics, IEEE Transactions on, vol. 57, no. 8, pp. 2700 -2708, aug. 2010.

[8] R. Peuget, S. Courtine, and J. Rognon, "Fault detection and isolation on a pwm inverter by knowledge-based model," in Industry Applications Conference, 1997. Thirty-Second IAS Annual Meeting, IAS '97., Conference Record of the 1997 IEEE, vol. 2, oct 1997, pp. $1471-1478$ vol.2.

[9] K. Rothenhagen and F. Fuchs, "Performance of diagnosis methods for igbt open circuit faults in three phase voltage source inverters for ac variable speed drives," in Power Electronics and Applications, 2005 European Conference on, 0-0 2005, pp. 10 pp. -P.7.

[10] R. de Araujo Ribeiro, C. Jacobina, E. da Silva, and A. Lima, "Fault detection of open-switch damage in voltage-fed pwm motor drive systems," Power Electronics, IEEE Transactions on, vol. 18, no. 2, pp. 587 - 593, mar 2003.

[11] P. Lezana, R. Aguilera, and J. Rodriguez, "Fault detection on multicell converter based on output voltage frequency analysis," Industrial Electronics, IEEE Transactions on, vol. 56, no. 6, pp. 2275 -2283, june 2009.

[12] S. Khomfoi and L. Tolbert, "Fault diagnosis and reconfiguration for multilevel inverter drive using ai-based techniques," Industrial Electronics, IEEE Transactions on, vol. 54, no. 6, pp. $2954-2968$, dec. 2007.

[13] V. Utkin, J. Guldner, and J. Shi, Sliding Mode Control in ElectroMechanical Systems, ser. Automation and Control Engineering. CRC Press, 2009.

[14] J.-J. E. Slotine, J. K. Hedrick, and E. A. Misawa, "On sliding observers for nonlinear systems," in American Control Conference, 1986, june 1986, pp. $1794-1800$.

[15] U. V. Haskara, I., "On sliding mode observers via equivalent control approach," International Journal of Control, vol. 71, no. 6, pp. 1051 1067, 1998.

[16] M. Almaleki, "Sliding mode observation of capacitor voltage in multilevel power converters," Ph.D. dissertation, The Unversity of Nottingham, 2010.

[17] A.-M. Lienhardt, G. Gateau, and T. Meynard, "Digital sliding-mode observer implementation using fpga," Industrial Electronics, IEEE Transactions on, vol. 54, no. 4, pp. $1865-1875$, aug. 2007.

[18] M. Almaleki, P. Wheeler, and J. Clare, "Sliding mode observation of capacitor voltage in multilevel power converters," in Power Electronics, Machines and Drives (PEMD 2010), 5th IET International Conference on, april 2010, pp. $1-6$. 